\title{
ON POLYNOMIAL EXTENSIONS OF RINGS
}

\author{
MICHIO YOSHIDA
}

Let $A$ be a commutative ring with unit element, and let $A[x]$ be a ring of polynomials in an indeterminate $x$ with coefficients in $A$. There are a number of well-known properties which $A$ shares with $A[x]$. We shall state one of them in the following.

Theorem. If $A$ is an integrally closed integral domain, then so also is $A[x]$.

In an earlier volume of this journal, Messrs. Butts, Hall and Mann (1) gave a proof of the theorem. The purpose of the present note is to give a simpler elementary proof and another valuation-theoretic one.

First proof. Let $K$ be the quotient field of $A$. At first we assume that $K$ is algebraically closed. If $f(x) \in K(x)$ is integral over $A[x]$, then $f(x) \in K[x]$, since $K[x]$ is integrally closed. Since $f(x)$ satisfies a monic equation with coefficients in $A[x]$, for any element $\xi$ in $A, f(\xi)$ is integral over $A$, that is, $f(\xi) \in A$. Set

$$
f(x)=a_{0}+a_{1} x+\ldots+a_{m} x^{m} \quad\left(a_{i} \in K\right),
$$

and take $m+1$ distinct elements $\xi_{j}(0 \leqslant j \leqslant m)$ in $A$. Then

$$
a_{0}+a_{1} \xi_{j}+\ldots+a_{m} \xi_{j}^{m}=\zeta_{j}, \quad \text { with } \zeta_{j} \in A(0 \leqslant j \leqslant m) .
$$

We solve these equations with respect to $a_{0}, a_{1}, \ldots, a_{m}$ and obtain

$$
a_{i}=\frac{\eta_{i}}{D} \text {, where } \eta_{i} \in A, D=\prod_{i<j}\left(\xi_{i}-\xi_{j}\right) \text {. }
$$

Here we notice that $\xi_{j}(0 \leqslant j \leqslant m)$ can be chosen such that $D=1$, since any monic equation of the form $\left(x-\xi_{1}\right)\left(x-\xi_{2}\right) \ldots\left(x-\xi_{k}\right)=1$, with $\xi_{i} \in A$, has a solution in $A$.

We now turn to the case in which $K$ is not algebraically closed. Let $\bar{K}$ be the algebraic closure of $K$ and let $\bar{A}$ be the integral closure of $A$ in $\bar{K}$. Then the integral closure of $A[x]$ in $K(x)$ is contained in $K(x) \cap \bar{A}[x]=A[x]$.

Second proof. We first recall the following fact. Let $\nu$ be a valuation of a field $K$, then $\nu$ can be extended to a valuation $\bar{\nu}$ of $K(x)$ by setting, for any polynomial

$$
f(x)=a_{0}+a_{1} x+\ldots+a_{m} x^{m}
$$

in $K[x]$,

$$
\bar{v}[f(x)]=\min _{0 \leqslant i \leqslant m} \nu\left(a_{i}\right)
$$

Received June 15, 1955. 
Since $A$ is integrally closed, $A$ can be represented as an intersection of a set of valuation rings of $K: A=\cap R_{\iota}$. Denote by $\bar{R}_{\iota}$ the valuation ring of $K(x)$, which is uniquely deduced from $R_{\imath}$ in the manner described just above. Then we have obviously

$$
A[x]=\cap \bar{R}_{\imath} \cap K[x] .
$$

Since $\bar{R}_{\iota}$ and $K[x]$ are integrally closed, so is $A[x]$.

Remark. An element $a$ of $K$ is said to be almost integral over $A$, if there exists an element $b \neq 0$ of $A$ such that $b a^{n} \in A$ for all $n$. If any element of $K$ which is almost integral over $A$ is contained in $A, A$ is said to be fully integrally closed. We note that in our theorem the phrase "integrally closed" can be replaced by "fully integrally closed." This can be proved as follows.

Let $f(x) \in K(x)$ be almost integral over $A[x]$, so that $f(x) \in K[x]$. We shall show that $f(x) \in A[x]$, by induction with respect to the degree of $f(x)$. By the definition of "almost integral," there exists a non-zero polynomial $g(x)$ in $A[x]$, such that

$$
g(x) f(x)^{\nu} \in A[x]
$$

for any positive integer $\nu$. Let $b, a$ be the leading coefficients of $g(x)$ and $f(x)$ respectively, and put $f(x)=a x^{m}+f_{1}(x)$. Then $b a^{v} \in A$, hence $a \in A$; consequently

$$
g(x) f_{1}(x)^{\nu}=g(x)\left[f(x)-a x^{m}\right]^{\nu} \in A[x],
$$

whence by the induction assumption, $f_{1}(x) \in A[x]$, hence $f(x) \in A[x]$.

\section{REFERENCES}

1. H. Butts, M. Hall and H. B. Mann, On integral closure, Can. J. Math., 6 (1954), 471-473.

\section{Hiroshima University}

